\title{
Reduced Complexity ML Detection for Differential Unitary Space-Time Modulation with Carrier Frequency Offset
}

\author{
Feifei Gao $\dagger$, A. Nallanathan $\ddagger$, and C. Tellambura $\S$ \\ $\dagger$ Institute for Infocomm Research, 1 Fusionopolis Way, \#21-01 Connexis, Singapore 138632 \\ Email: feifeigao@ieee.org \\ $\ddagger$ Division of Engineering, Kings College London, London WC2R 2LS, U.K. \\ Email: arumugam.nallanathan@kcl.ac.uk \\ $\S$ Department of Electrical and Computer Engineering, University of Alberta, Edmonton, AB T6G 2V4, Canada \\ Email: chintha@ece.ualberta.ca
}

\begin{abstract}
Recently, a maximum likelihood (ML) detection rule for differential unitary space time modulation (DUSTM) under the existence of unknown carrier frequency offset (CFO) has been derived. However, the ML detection is based on the exhaustive search over all the unitary group codes. In this paper, we design an efficient detection algorithm for newly derived ML rule, by modifying the bound intersection detector (BID). Our proposed algorithm is seen as a generalization of the existing BID that is known to be an optimal detector for the conventional DUSTM. The simulation results show that the proposed algorithm can save a large portion of the computational complexity compared to the naive searching method.
\end{abstract}

\section{INTRODUCTION}

Most differential space time coding (DSTC) works [1][3] assume perfect synchronization while focussing on either the code design or the receiver design only. However, the performance of DSTC degrades greatly if the carrier frequency offset (CFO) is not compensated for at the receiver before symbol detection. Conventional ways using training to estimate CFO [4], [5] should not be used since this method clearly contradicts the original motivation of using DSTC.

Recently, Liu [6] and Ma [7] proposed a new modulation scheme that can handle both unknown channel and CFO. This method is based on unitary matrix group [2], [3] and uses time-domain double differential modulation, where the first differentiation is to remove the unknown channel and the second differentiation is to remove the unknown CFO. Throughout this paper, we will name the original differential scheme proposed in [2], [3] as differential unitary space time modulation (DUSTM) while name the double differential scheme in [6], [7] as double differential unitary space time modulation (DDUSTM).

Although DDUSTM successfully deals with CFO, it is still of interest to investigate whether the conventional DUSTM can handle the unknown CFO, itself. The affirmative answer was given in [8] recently, where it is shown that by sacrificing one degree of freedom in transmit antennas, the ML symbol detector can be derived for DUSTM even with the unknown
CFO. The resulting algorithm is named, correspondingly, as modified DUSTM (MDUSTM).

The ML detection for MDUSTM requires search over the entire signal space whose complexity increases exponentially with the product of the transmission rate and the number of the transmit antennas. To avoid the naive exhaustive search, we generalize the BID [9], [10] to optimally detect MDUSTM, resulting in the reduction of the detection complexity.

Notation: Vectors and matrices are represented in boldface small and capital letters; the transpose, Hermitian, and inverse of matrix $\mathbf{A}$ are denoted by $\mathbf{A}^{T}, \mathbf{A}^{H}$, and $\mathbf{A}^{-1}$, respectively; $\operatorname{tr}(\mathbf{A})$ and $\|\mathbf{A}\|_{F}$ are the trace and the Frobenius norm of $\mathbf{A}$; $\operatorname{diag}\{\mathbf{a}\}$ denotes a diagonal matrix with the diagonal element constructed from $\mathbf{a} ; \operatorname{gcd}(a, b)$ is the greatest common divisor of $a$ and $b ;|x|$ is the absolute value of a scalar $x ;\lfloor x\rfloor$ denotes the closest integer to $x$ to minus infinity, and the imaginary unit $\mathrm{j}=\sqrt{-1}$.

\section{SySTEM MOdEL}

Consider a MIMO system with $N_{t}$ transmit and $N_{r}$ receive antennas over a Rayleigh flat fading environment. Each time slot occupies an interval of $T_{s}$ seconds, and each block consists of $N_{t}$ time slots. The transmitted signals are modulated by choosing a unitary matrix from a finite group $\mathcal{V}=\left\{\mathbf{V}_{l}, l=\right.$ $0,1, \ldots, L-1\}$, where $L=2^{N_{t} R}$, and $R$ denotes the data rate. As in [6] and [7], we consider diagonal constellations based on the cyclic group whose unitary matrices $\mathbf{V}_{l}$ are written as

$$
\mathbf{V}_{l}=\operatorname{diag}\left\{e^{\mathrm{j} 2 \pi u_{1} l / L}, e^{\mathrm{j} 2 \pi u_{2} l / L}, \ldots, e^{\mathrm{j} 2 \pi u_{N_{t}} l / L}\right\},
$$

where $u_{i}$ are integers and can be optimized according to the upper bound of the pairwise error probability (PEP) [2]. The transmitted symbol during the $k$ th block is denoted by the $N_{t} \times N_{t}$ matrix

$$
\mathbf{S}[k]=\mathbf{V}[k] \mathbf{S}[k-1],
$$

and the $(t, i)$ th element of $\mathbf{S}[k]$ is transmitted from the $i$ th antenna in the $t+(k-1) N_{t}$ time slot. The initial transmission matrix $\mathbf{S}[0]$ can be chosen as an arbitrary matrix from $\mathcal{V}$. 
The received signals from all $N_{r}$ antennas through time slot $1+(k-1) N_{t}$ to $k N_{t}$ may be expressed in an $N_{t} \times N_{r}$ matrix form

$$
\mathbf{X}[k]=\sqrt{\rho} \mathbf{S}[k] \mathbf{H}[k]+\mathbf{W}[k],
$$

where $\rho$ is the received signal to noise ratio (SNR) and $\mathbf{W}[k]$ is the $N_{t} \times N_{r}$ noise matrix whose entries are complex Gaussian noise with unit variance and are independent across the receiver and time indices. The $(i, j)$ th entry of $\mathbf{H}[k]$, denoted by $h_{i, j}[k]$, is the channel gain from the $i$ th transmit antenna to the $j$ th receive antenna, which remains constant during one block interval. All path gains are assumed statistically independent $\left(E\left\{h_{i, j}[k] h_{i^{\prime}, j^{\prime}}^{*}[k]\right\}=\delta\left(i-i^{\prime}\right) \delta\left(j-j^{\prime}\right)\right)$ with unit variances.

Suppose the CFO is constant in one block but may vary from block to block due to the CFO drifting [7]. Let $\varepsilon_{k}$ denote the normalized CFO by the sampling period $T_{s}$ during the $k$ th block. Then, the phase distortion on the $i+(k-1) N_{t}$ time slot is

$$
\theta_{i, k}=2 \pi \varepsilon_{k}, \quad i=1, \ldots, N_{t}, k=0,1, \ldots
$$

Therefore, the overall phase rotation before the $i+(k-1) N_{t}$ time slot, denoted as $\vartheta_{i, k}$, is that accumulated from all the previous time slots:

$$
\vartheta_{i, k}=\sum_{\tau<i} \sum_{c<k} \theta_{\tau, c}=2 \pi N_{t} \sum_{c=0}^{k-1} \varepsilon_{c}+2 \pi(i-1) \varepsilon_{k} .
$$

The received signal block $\mathbf{X}[k]$ with the unknown CFO is then modeled as

$$
\mathbf{X}[k]=\sqrt{\rho} e^{j \vartheta_{1, k}} \boldsymbol{\Gamma}\left(\varepsilon_{k}\right) \mathbf{S}[k] \mathbf{H}[k]+\mathbf{W}[k],
$$

where $\boldsymbol{\Gamma}\left(\varepsilon_{k}\right)$ is the diagonal matrix with the form

$$
\boldsymbol{\Gamma}\left(\varepsilon_{k}\right)=\operatorname{diag}\left\{1, e^{\mathrm{j} 2 \pi \varepsilon_{k}}, \ldots, e^{\mathrm{j} 2 \pi\left(N_{t}-1\right) \varepsilon_{k}}\right\} .
$$

From [8], the ML detection (MLD) of $\mathbf{V}[k]$ after receiving $\mathbf{X}[k-1]$ and $\mathbf{X}[k]$ is

$$
\hat{\mathbf{V}}[k]=\arg \max _{\mathbf{V}[k]}\left|\operatorname{tr}\left(\mathbf{V}[k] \mathbf{X}[k-1] \mathbf{X}^{H}[k]\right)\right| .
$$

Note that, the MLD (8) is not affected by the value of the CFO. The detection of $\mathbf{V}[k]$ by solving (8) requires an exhaustive search over the whole constellation $\mathcal{V}$. The complexity of this naive algorithm increases exponentially with the increase of the transmission rate or the number of the transmit antennas, and prohibits the use of this algorithm in practice.

\section{EfFicient Detection Algorithms}

\section{A. Problem Formulation}

In this section, we adapt the BID proposed in [9] for DUSTM to our proposed MLD (8). Denote the $(i, i)$-th entry of $\mathbf{X}[k-1] \mathbf{X}^{H}[k]$ as $d_{i}$. The MLD (8) is equivalent to

$$
\begin{aligned}
\hat{l} & =\arg \max _{l}\left|\sum_{i=1}^{N_{t}} d_{i} e^{\mathrm{j} 2 \pi u_{i} l / L}\right| \\
& =\arg \max _{l} \sum_{i=1}^{N_{t}} \sum_{j=1}^{N_{t}} d_{i} d_{j}^{*} e^{\mathrm{j} 2 \pi u_{i} l / L} e^{-\mathrm{j} 2 \pi u_{j} l / L} \\
& =\arg \min _{l} \sum_{i=1}^{N_{t}-1} \sum_{j=i+1}^{N_{t}}-2 \Re\left\{d_{i} d_{j}^{*} e^{\mathrm{j} 2 \pi u_{i} l / L} e^{-\mathrm{j} 2 \pi u_{j} l / L}\right\} \\
& =\arg \min _{l} \sum_{i=1}^{N_{t}-1} \sum_{j=i+1}^{N_{t}} A_{i, j}-B_{i, j} \cos \left(\left(u_{i, j} l-\phi_{i, j}\right) 2 \pi / L\right) \\
& =\arg \min _{l} \lambda(l),
\end{aligned}
$$

where

$$
\begin{aligned}
A_{i, j} & =\left|d_{i}\right|^{2}+\left|d_{j}\right|^{2}, & B_{i, j} & =2\left|d_{i} d_{j}\right|, \\
u_{i, j} & =u_{j}-u_{i}, & \phi_{i, j} & =\angle\left(d_{i} / d_{j}\right) L / 2 \pi,
\end{aligned}
$$

and $\angle\left(d_{i} / d_{j}\right)$ is the phase of $d_{i} / d_{j}$. Noted that each summand in (9) is non-negative. Therefore, a sphere decoding [12] like process can be considered to reduce the complexity of the detection, where, instead of searching all of the $0 \leq l<L$, we can find an initial radius $C$ and search only the values of $l$ subjected to $\lambda(l)<C$. The initial radius $C$ is selected such that the probability $P\left(\lambda\left(l_{0}\right)<C\right)$ is no less than $\epsilon$.

\section{B. Statistics of the Initial Radius}

From (9), we have the following equation:

$$
\lambda(l)=N_{t} \sum_{i=1}^{N_{t}}\left|d_{i}\right|^{2}-\left|\operatorname{tr}\left(\mathbf{V}_{l}[k] \mathbf{X}[k-1] \mathbf{X}^{H}[k]\right)\right|^{2} .
$$

Since $N_{t} \sum_{i=1}^{N_{t}}\left|d_{i}\right|^{2}$ is directly obtained from the received signal, we could consider the statistics of the second term only. For the true solution $l_{0},\left|\operatorname{tr}\left(\mathbf{V}_{l} \mathbf{X}[k-1] \mathbf{X}^{H}[k]\right)\right|$ could be represented by

$$
\left|\operatorname{tr}\left((\sqrt{\rho} \mathbf{H}+\tilde{\mathbf{W}}[k])^{H}(\sqrt{\rho} \mathbf{H}+\tilde{\mathbf{W}}[k-1])\right)\right|,
$$

where

$$
\begin{aligned}
& \tilde{\mathbf{W}}[k-1]=e^{-\mathrm{j} \vartheta_{1, k-1}} \boldsymbol{\Gamma}^{H}(\varepsilon) \mathbf{S}^{H}[k-1] \mathbf{W}[k], \\
& \tilde{\mathbf{W}}[k]=e^{-\mathrm{j}\left(\vartheta_{1, k-1}+2 \pi N_{t} \varepsilon\right)} \boldsymbol{\Gamma}^{H}(\varepsilon) \mathbf{S}^{H}[k-1] \mathbf{V}_{l}^{H} \mathbf{W}[k-1] .
\end{aligned}
$$

and is independent from both code group $\mathcal{V}$ and the CFO $\varepsilon$. Note that, the radius $\lambda\left(l_{0}\right)$ corresponding to the true solution $l_{0}$ is related to SNR in the algorithm here.

Similar to [12], we first need to find the probability density function (PDF) function of $\lambda\left(l_{0}\right)$. At the high SNR region, the second-order noise term can be ignored and then (11) becomes

$$
|\underbrace{\sum_{i=1}^{N_{t}} \sum_{j=1}^{N_{r}}\left(\rho\left|h_{i j}\right|^{2}+\sqrt{\rho} h_{i j}^{*} \tilde{w}_{i j}[k-1]+\sqrt{\rho} h_{i j} \tilde{w}_{i j}^{*}[k]\right)}_{x}| .
$$


Conditioned on a specific realization of $\mathbf{H}, x$ is a Gaussian random variable and could be used to approximate $\operatorname{tr}\left(\mathbf{V}_{l} \mathbf{X}[k-\right.$ 1] $\left.\mathbf{X}^{H}[k]\right)$. Consequently, $\chi=|x|^{2}$ is a non-central chi-squared distributed random variable with mean $\rho\|\mathbf{H}\|_{F}^{2}$ and variance $\rho\|\mathbf{H}\|_{F}^{2}$ per dimension. The conditioned PDF of $\chi$ is then expressed as

$$
p_{\chi}(\chi \mid \mathbf{H})=\frac{1}{2 \rho\|\mathbf{H}\|_{F}^{2}} e^{-\frac{\rho^{2}\|\mathbf{H}\|_{F}^{4}+\chi}{2 \rho\|\mathbf{H}\|_{F}^{2}}} I_{0}(\sqrt{\chi}), \quad \chi \geq 0,
$$

where $I_{\nu}(\cdot)$ is the $\nu$ th order modified Bessel functions of the first kind. As well, $\iota=\|\mathbf{H}\|_{F}^{2}$ is a chi-square random variable with degree $2 N_{t} N_{r}$ whose PDF is

$$
p_{\iota}(\iota)=\frac{2^{N_{t} N_{r}}}{\left(N_{t} N_{r}-1\right) !} \iota^{N_{t} N_{r}-1} e^{-\iota}, \quad \iota \geq 0 .
$$

Therefore, $p_{\chi}(\chi)$ can be calculated as

$$
\begin{aligned}
p_{\chi}(\chi) & =\int_{0}^{\infty} p_{\chi}(\chi \mid \mathbf{H}) p_{\iota}(\iota) d \iota \\
& =\frac{2^{N_{t} N_{r}-1} I_{0}(\sqrt{\chi})}{\rho\left(N_{t} N_{r}-1\right) !} \int_{0}^{\infty} \iota^{N_{t} N_{r}-2} e^{-\left(\frac{\rho}{2}+1\right) \iota-\frac{\chi}{2 \rho \iota}} d \iota .
\end{aligned}
$$

From [13, pp. 384, and pp.364 ], the above PDF could be written into a closed form as

$$
\begin{aligned}
& p_{\chi}(\chi)= \\
& \begin{cases}\frac{2^{N_{t} N_{r}}}{\rho\left(N_{t} N_{r}-1\right) !}\left(\frac{\chi}{\rho^{2}+2 \rho}\right)^{\frac{N_{t} N_{r}-1}{2}} & \\
\quad \times I_{0}(\sqrt{\chi}) K_{\left(N_{t} N_{r}-1\right)}\left(2 \sqrt{\left(\frac{1}{4}+\frac{1}{2 \rho}\right) \chi}\right), & \chi>0, \\
\frac{2^{N_{t} N_{r}-1}}{\rho\left(N_{t} N_{r}-1\right)}\left(\frac{\rho}{2}+1\right)^{1-N_{t} N_{r}}, & \chi=0 .\end{cases}
\end{aligned}
$$

where $K_{\nu}(\cdot)$ is the $\nu$ th order modified Bessel functions of the second kind. One observation is that $p_{\chi}(\chi)$ is related to $N_{t} N_{r}$ and SNR only. Then the cumulative distribution function (CDF) of $\chi$, denoted as $F_{\chi}(\chi)$, can be expressed as

$$
F_{\chi}(\chi)=\int_{0}^{\chi} p_{\chi}(t) d t
$$

The CDF of both $\left|\operatorname{tr}\left(\mathbf{V}_{l} \mathbf{X}[k-1] \mathbf{X}^{H}[k]\right)\right|^{2}$ and $|x|^{2}$ are shown in Fig. 1 for different pairs of $\left(N_{t}, N_{r}\right)$ at $\mathrm{SNR}=0 \mathrm{~dB}$. The statistics of $|x|^{2}$ fits that of $\left|\operatorname{tr}\left(\mathbf{V}_{l} \mathbf{X}[k-1] \mathbf{X}^{H}[k]\right)\right|^{2}$ very well even at a low SNR. Finally, the CDF of the radius $\lambda\left(l_{0}\right)$ is given by

$$
F_{\lambda}(C)=P\left(\lambda\left(l_{0}\right)<C\right)=1-F_{\chi}\left(N_{t} \sum_{i=1}^{N_{t}}\left|d_{i}\right|^{2}-C\right) .
$$

Then, the initial radius $C$ should be designed from

$$
F_{\chi}\left(N_{t} \sum_{i=1}^{N_{t}}\left|d_{i}\right|^{2}-C\right) \leq 1-\epsilon
$$

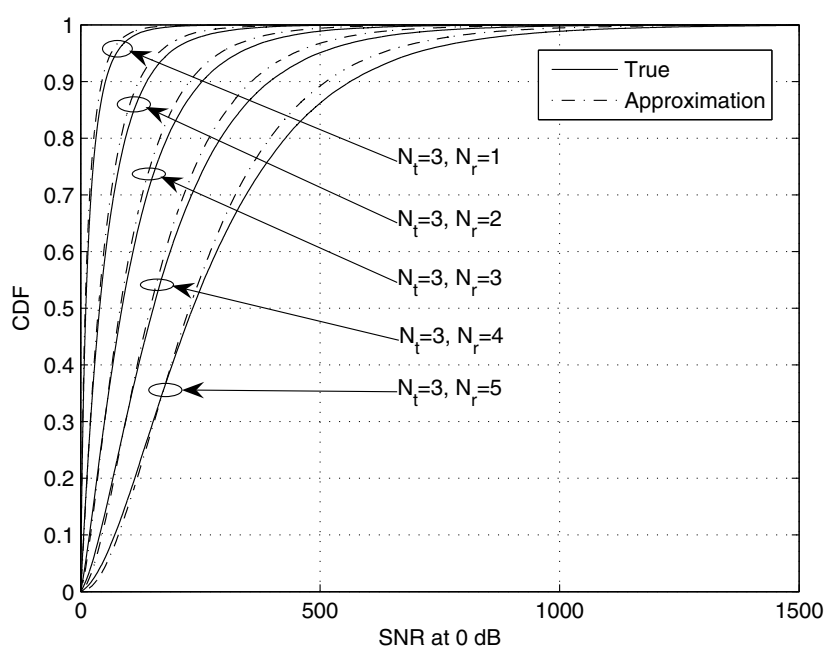

Fig. 1. The CDFs of $|x|^{2}$ and $\left|\operatorname{tr}\left(\mathbf{V}_{l} \mathbf{X}[k-1] \mathbf{X}^{H}[k]\right)\right|^{2}$ at $\mathrm{SNR}=0 \mathrm{~dB}$.

\section{The Algorithm}

To find all the $l$ 's that satisfy $\lambda(l)<C$, we note that $\lambda(l)$ in (9) consists of $\frac{N_{t}\left(N_{t}-1\right)}{2}$ non-negative terms. Thus, a necessary condition for $\lambda(l)<C$ is that each term of (9) is less than $C$, or equivalently

$$
\begin{array}{r}
A_{i, j}-B_{i, j} \cos \left(\left(\bmod \left(u_{i, j} l, L\right)-\phi_{i}\right) 2 \pi / L\right)<C, \\
1 \leq i<j \leq N_{t},
\end{array}
$$

where $\bmod (x, L)$ reduces $x$ to an integer between 0 and $L$. Let us define the candidate set $\mathcal{L}_{i, j}=\left\{l \mid A_{i, j}-\right.$ $\left.B_{i, j} \cos \left(\left(\bmod \left(u_{i, j} l, L\right)-\phi_{i}\right) 2 \pi / L\right)<C, 0 \leq l<L\right\}$. To find $\mathcal{L}_{i, j}$, we first show how, given a number $n$, to find an $l$ such that $\bmod \left(u_{i, j} l, L\right)=n$. In [9], an efficient algorithm by using the well-known Extended Euclidean Algorithm [14] is given by considering the fact that $u_{i, j}$ and $L$ are coprime. However, for MDUSTM, the optimal $u_{i, j}$ and $L$ may not be coprime [8]. Let $\xi_{i, j}=\operatorname{gcd}\left(u_{i, j}, L\right), u_{i, j}^{\prime}=u_{i, j} / \xi_{i, j}$, and $L^{\prime}=L / \xi_{i, j}$. Clearly, we have $\bmod \left(u_{i, j} l, L\right)=\xi_{i, j}$. $\bmod \left(u_{i, j}^{\prime} l, L^{\prime}\right)=n$. Therefore, there exists such an $l$ if and only if $\bmod \left(n, \xi_{i, j}\right)=0$. If $\bmod \left(n, \xi_{i, j}\right)=0$ holds, let $n^{\prime}=n / \xi_{i, j}$. Since now $u_{i, j}^{\prime}$ is relatively prime to $L^{\prime}$, the gcd of $u_{i, j}^{\prime}$ and $L^{\prime}$ is 1 . The Extended Euclidean Algorithm computes the gcd of $u_{i, j}^{\prime}$ and $L^{\prime}$, as well as the numbers $\mu_{i, j}$ and $\kappa$ such that

$$
u_{i, j}^{\prime} \mu_{i, j}+\kappa L^{\prime}=1,
$$

where 1 is the gcd of $u_{i, j}^{\prime}$ and $L^{\prime}$. For the details of the Extended Euclidean Algorithm, see [14]. To find $\bmod \left(u_{i, j}^{\prime} l, L^{\prime}\right)=n^{\prime}$, we multiply both sides of (23) by $n^{\prime}$, to obtain

$$
u_{i, j}^{\prime}\left(n^{\prime} \mu_{i, j}\right)+\kappa n^{\prime} L^{\prime}=n^{\prime} .
$$

Therefore, $\tilde{l}=\bmod \left(n^{\prime} \mu_{i, j}, L^{\prime}\right)$ satisfies $\bmod \left(u_{i, j}^{\prime} \tilde{l}, L^{\prime}\right)=n^{\prime}$, or, equivalently, $\bmod \left(u_{i, j} \tilde{l}, L\right)=n$. Since $0 \leq \tilde{l}<L^{\prime}$, a total of $\xi_{i, j}$ different $l$ satisfy $\bmod \left(u_{i, j} l, L\right)=n$ (They are 


$$
\{\hat{\varepsilon}, \hat{\mathbf{V}}[k]\}=\arg \max _{\varepsilon, \overline{\mathbf{V}}[k]} \sum_{i=0}^{N-1} \sum_{j=i+1}^{N} b_{i, j} \Re\left\{e^{\mathrm{j} 2 \pi N_{t}(j-i) \varepsilon} \operatorname{tr}\left(\left(\prod_{m=i+k}^{j+k-1} \mathbf{V}[m]\right) \mathbf{X}[i+k-1] \mathbf{X}^{H}[j+k-1]\right)\right\} .
$$

$$
\hat{\overline{\mathbf{V}}}[k]=\arg \max _{\overline{\mathbf{V}}[k]} \sum_{i=0}^{N-1} \sum_{j=i+1}^{N} b_{i, j}\left|\operatorname{tr}\left(\left(\prod_{m=i+k}^{j+k-1} \mathbf{V}[m]\right) \mathbf{X}[i+k-1] \mathbf{X}^{H}[j+k-1]\right)\right| .
$$

$$
\hat{\mathbf{V}}[k]=\arg \max _{\overline{\mathbf{V}}[k]} \sum_{i=0}^{N-1} \sum_{j=i+1}^{N} b_{i, j}\left|\operatorname{tr}\left(\left(\prod_{m=i+k}^{j+k-1} \mathbf{V}[m]\right) \mathbf{X}[i+k-1] \mathbf{X}^{H}[j+k-1]\right)\right|^{2} .
$$

$l=\tilde{l}+k L^{\prime}, k=0, \ldots, \xi_{i, j}-1$, respectively). We can now determine $\mathcal{L}_{i, j}$. Define

$$
U B_{i, j}=\left\lfloor\phi_{i}+\rho_{i}\right\rfloor, \quad L B_{i, j}=\left\lceil\phi_{i}-\rho_{i}\right\rceil,
$$

where

$$
\rho_{i, j}=\frac{L}{2 \pi} \cos ^{-1}\left(\frac{A_{i, j}-C}{B_{i, j}}\right) .
$$

Let $\mathcal{S}_{i, j}=\left\{n / \xi_{i, j} \mid n \in \mathbb{Z}, L B_{i} \leq n \leq\right.$ $U B_{i}$, and $\left.\bmod \left(n, \xi_{i, j}\right)=0\right\}$. Following the same arguments as in [9], we find $\mathcal{L}_{i, j}$ is given by

$$
\mathcal{L}_{i, j}=\bigcup_{0 \leq k<\xi_{i, j}}\left(\bmod \left(\mu_{i, j} \mathcal{S}_{i, j}, L^{\prime}\right)+k L^{\prime}\right), 1 \leq i<j \leq N_{T},
$$

where both the mod operation and addition are performed component-wise. The candidate set for all $l$ 's such that $\lambda(l)<$ $C$ is the intersection of all of the $\frac{N_{t}\left(N_{t}-1\right)}{2}$ sets $\mathcal{L}_{i, j}$ is

$$
\mathcal{L}=\bigcap_{1 \leq i<j \leq N_{T}} \mathcal{L}_{i, j}
$$

As with the BID in [9], we first choose $l^{*}$ from $\mathcal{L}$. $C$ is then replaced by the new cost $\lambda\left(l^{*}\right)$, and $l^{*}$ is deleted from the set $\mathcal{L}\left(\mathcal{L}=\mathcal{L}-\left\{l^{*}\right\}\right)$. All $\mathcal{L}_{i, j} 1 \leq i<j \leq N_{T}$ are updated by using the new bound $C$. In later iterations, (28) is replaced by

$$
\mathcal{L}=\mathcal{L} \cap\left(\bigcap_{1 \leq i<j \leq N_{T}} \mathcal{L}_{i, j}\right)
$$

The process continues until $\mathcal{L}$ becomes the null set. The $l$ with the minimum cost is then the optimal solution. This optimal detection algorithm is called the "modified BID."

If $u_{i, j}$ is coprime to $L$ or $\xi_{i, j}=1$, the modified BID reduces to the original BID [9]. Alternatively, (8) can also be suboptimally solved by using the lattice reduction algorithm proposed in [15] for the detection of DUSTM.

\section{Multiple Symbol Detection and the Efficient ALGORITHM}

\section{A. Multiple Symbol Detection}

If CFO remains constant across more than two blocks or varies very slowly among several blocks, multiple symbol detection (MSD) can also be applied for DUSTM as in [9], [11]. In MSD, the transmitted symbols in $N$ consecutive intervals are estimated using $N+1$ received blocks. Let us define $\overline{\mathbf{V}}[k]=\left[\mathbf{V}^{H}[k], \ldots, \mathbf{V}^{H}[k+N-1]\right]^{H}$. By using the same approach as in [9], [11], the joint ML estimates of $\overline{\mathbf{V}}[k]$ and $\varepsilon$ can be derived as in (30), where $b_{i, j}$ is the $(i, j)$-th entry of $\mathbf{B}=\left(\mathbf{C}_{h}+\sigma_{n}^{2} \mathbf{I}_{N+1}\right)^{-1}$, and

$$
\mathbf{C}_{h}=\left[\begin{array}{cccc}
\varphi_{h}[0] & \varphi_{h}[1] & \cdots & \varphi_{h}[N] \\
\varphi_{h}[-1] & \varphi_{h}[0] & \vdots & \vdots \\
\vdots & \vdots & \ddots & \vdots \\
\varphi_{h}[-N] & \cdots & \cdots & \varphi_{h}[0]
\end{array}\right]
$$

is the channel covariance matrix. Since the phase $(j-i) \varepsilon$ varies for different terms in (30), we only propose a suboptimal MSD as in (32). We denote (32) as MSD1 in the following. However, it is difficult to design efficient detection algorithms for (32) directly because the sum of absolute terms is difficult to handle in practice. As an approximation of (32), we propose a new estimation criterion as shown in (33) for designing efficient MSD algorithms. We denote (33) as MSD2.

\section{B. Efficient Algorithm}

For the MSD of the diagonal signals, the search space increases to $L^{N}$, and the computation of the metric (33) is more complex than in the SSD case. For cyclic group code shown in (1), we need to estimate $\hat{\mathbf{l}}=\left[\hat{l}_{k}, \hat{l}_{k+1}, \ldots, \hat{l}_{k+N-1}\right]$.

Denote the $(p, p)$ th entry of $\mathbf{X}[i+k-1] \mathbf{X}[j+k-1]$ as $d_{i j, p}$. The MLD (33) is equivalent to

$$
\begin{array}{r}
\hat{\mathbf{l}}=\arg \max _{\mathbf{l}} \sum_{i=0}^{N-1} \sum_{j=i+1}^{N} b_{i, j}\left|\sum_{p=1}^{N_{t}} d_{i j, p} e^{\mathrm{j} 2 \pi u_{p} \sum_{m=i+k}^{j+k-1} l_{m} / L}\right|^{2} \\
=\arg \max _{\mathbf{l}} \sum_{i=0}^{N-1} \sum_{j=i+1}^{N} \sum_{p=1}^{N_{t}} \sum_{q=1}^{N_{t}} b_{i, j} d_{i j, p} d_{i j, q}^{*} \\
\times e^{-\mathrm{j} 2 \pi u_{p, q} \sum_{m=i+k}^{j+k-1} l_{m} / L}
\end{array}
$$

Define $\psi_{i, j}=\angle b_{i, j}$ and $\tilde{d}_{i j, p} \triangleq\left|b_{i, j}\right|^{0.5} d_{i j, p}$. The MLD is 
further expressed as

$$
\begin{gathered}
\hat{\mathbf{l}}=\arg \max _{\mathbf{l}} \sum_{i=0}^{N-1} \sum_{j=i+1}^{N} \sum_{p=1}^{N_{t}} \sum_{q=1}^{N_{t}} \tilde{d}_{i j, p} \tilde{d}_{i j, q}^{*} \\
\times e^{-\mathrm{j}\left(2 \pi u_{p, q} \sum_{m=i+k}^{j+k-1} l_{m} / L-\psi_{i, j}\right)} \\
=\arg \min _{\mathbf{l}} \sum_{i=0}^{N-1} \sum_{j=i+1}^{N} \sum_{p=1}^{N_{t}-1} \sum_{q=p+1}^{N_{t}} \tilde{A}_{i j, p q} \\
-\tilde{B}_{i j, p q} \cos \left(\left(u_{p, q} \sum_{m=i+k}^{j+k-1} l_{m}-\tilde{\phi}_{i j, p q}\right)\right. \\
=\arg \min _{\mathbf{l}} \sum_{i=0}^{N-1} \sum_{j=i+1}^{N} \underbrace{(35)}_{\sum_{p=1}^{N_{t}-1} \sum_{q=p+1}^{N_{t}} \tilde{\lambda}_{p q}\left(\sum_{m=i+k}^{j+k-1} l_{m}^{j+k-1} l_{m}\right)},
\end{gathered}
$$

where

$$
\begin{aligned}
A_{i j, p q} & =\left|\tilde{d}_{i j, p}\right|^{2}+\left|\tilde{d}_{i j, q}\right|^{2}, \quad B_{i j, p q}=2\left|\tilde{d}_{i j, p} \tilde{d}_{i j, q}\right|, \\
\phi_{i j, p q} & =\angle\left(\tilde{d}_{i j, p} / \tilde{d}_{i j, q}+\psi_{i, j}\right) L / 2 \pi,
\end{aligned}
$$

Noted that the summand $\tilde{\lambda}_{p q}(\cdot)$ or $\tilde{\lambda}(\cdot)$ in (35) is non-negative. Therefore, similar idea can be applied here. First find a initial radius $C$. Then, we consider the summand $\tilde{\lambda}\left(l_{k+N-1}\right)$ which corresponds to $i=N-1$. For $\tilde{\lambda}\left(l_{k+N-1}\right)<C$, we can apply the SSD based modified BID algorithm, and find the candidate set $\tilde{\mathcal{L}}_{k+N-1}$ for all possible $\hat{l}_{k+N-1}$. For every $\hat{l}_{k+N-1}$ in $\tilde{\mathcal{L}}_{k+N-1}$, the bound can be improved to $C-B_{k+N-1}$, where $B_{k+N-1}=\tilde{\lambda}\left(\hat{l}_{k+N-1}\right)$. Then, we consider $\tilde{\lambda}\left(l_{k+N-2}+\right.$ $\left.\hat{l}_{k+N-1}\right)+\tilde{\lambda}\left(l_{k+N-2}\right)$, which corresponding to $i=N-2$. For $\tilde{\lambda}\left(l_{k+N-2}+\hat{l}_{k+N-1}\right)+\tilde{\lambda}\left(l_{k+N-2}\right)<C-B_{k+N-1}$, we can still apply the modified BID and find the candidate set $\tilde{\mathcal{L}}_{k+N-1}$ for $\hat{l}_{k+N-2}$. This process go on until $i=0$.

\section{Simulation Results}

In this section, the proposed modified BID algorithm is simulated. The average number of the flops is the figure of merit. The signal transmitted in the first block is chosen as $\mathbf{S}[0]=\mathbf{I}_{N_{t}}$ and channels are assumed constant during two consecutive blocks.

Fig. 2 - Fig. 4 shows the complexity of the modified BID in flops for different rates $R=1$ and $R=2$, under three cases: 1) $N_{t}=2$ and $N_{r}=2$;2) $N_{t}=3$ and $N_{r}=4$; 3) $N_{t}=4$ and $N_{r}=3$. The complexity exhibited includes only that from the detection process. We use the flops function in MATLAB for the modified BID (this function provides an estimate of the number of floating-point operations performed by a sequence of MATLAB statements), and the complexity of the ML algorithm is approximated by $2^{N_{t} R}\left(8 N_{t}+3\right)$ in (9). No parallelization is considered. The complexity of the modified BID is not reduced as quickly as that of the traditional BID [9] when SNR increases, because the radius $C$ also increases when the SNR becomes higher. For $R=1$, the modified BID can always save half the number of the flops compared with ML method. However, for $R=2$, the amount of the complexity saving reduces when the $N_{t}$ is getting larger. Nonetheless,

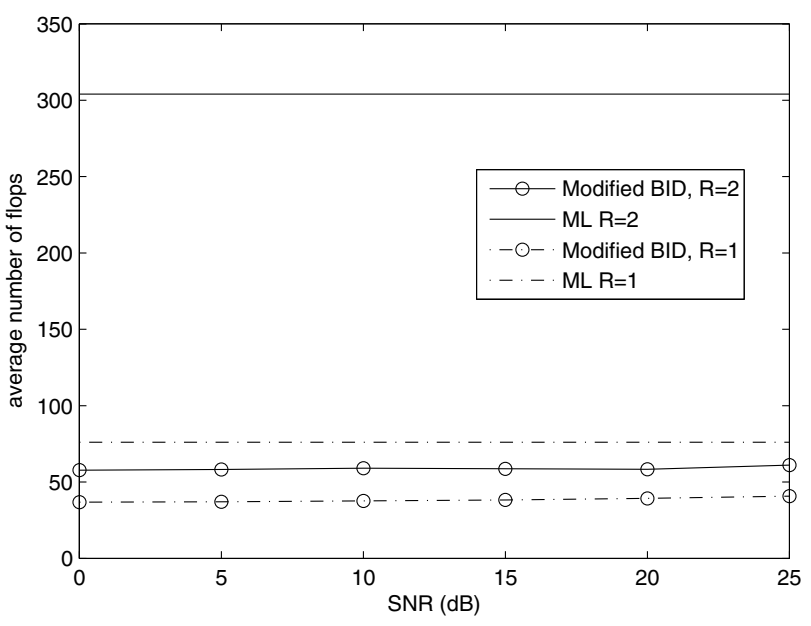

Fig. 2. Average number of flops versus SNR for $N_{t}=2, N_{r}=2$ with the rate $R=1$ and $R=2$.

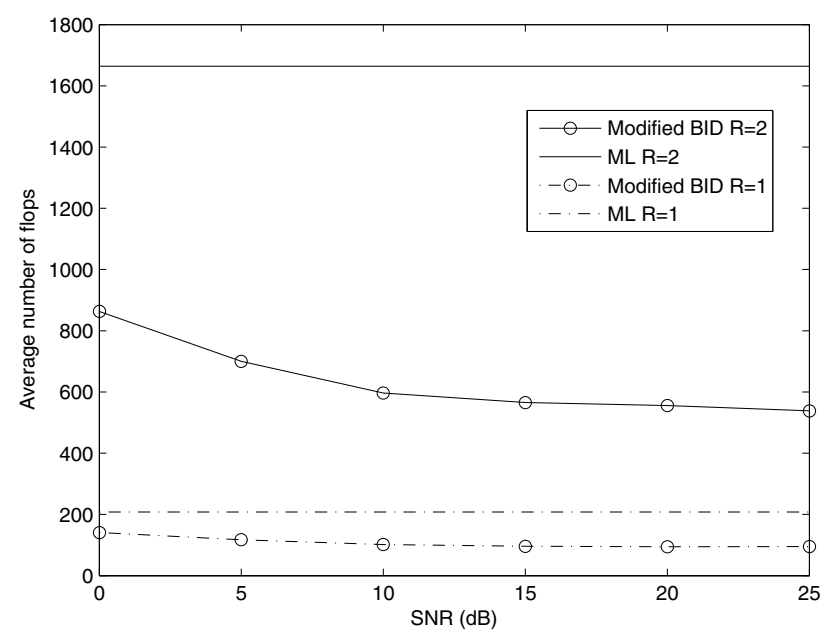

Fig. 3. Average number of flops versus SNR for $N_{t}=3, N_{r}=4$ with the rate $R=1$ and $R=2$.

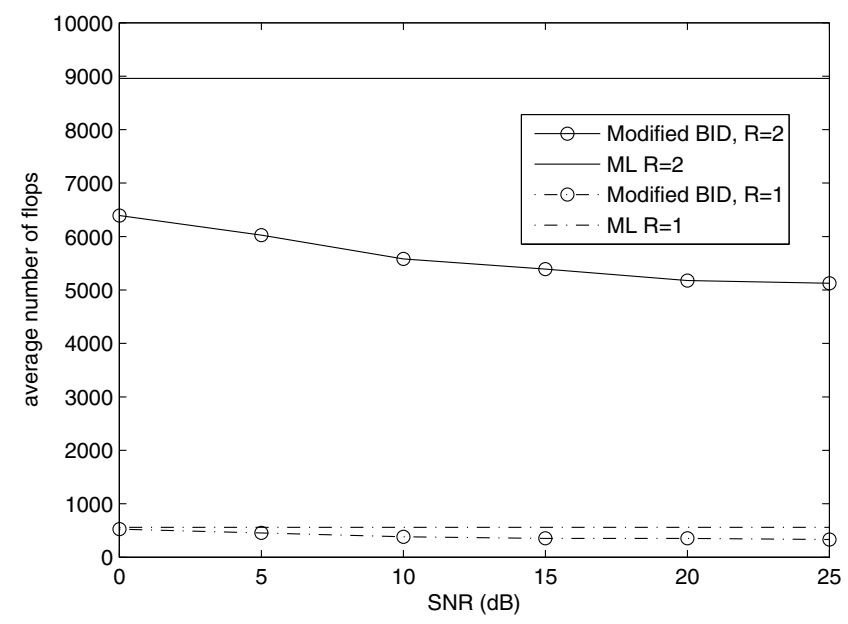

Fig. 4. Average number of flops versus SNR for $N_{t}=4, N_{r}=3$ with the rate $R=1$ and $R=2$. 
for regular system parameters, the use of the modified BID is more computationally efficient than that of the conventional ML searching.

\section{CONCLUSiOnS}

In this paper, we design a computationally efficient detection algorithm for the ML rules in MDUSTM scheme. The method uses the sphere decoding thinking where only the symbol constellations in a restrained circle are considered and compared. We determine the statistics of the initial radius that could guarantee the true ML detection with probability $\epsilon$. Finally, simulation results under different system parameters are conducted to show efficiency of the proposed algorithm.

\section{REFERENCES}

[1] V. Tarokh and H. Jafarkhani, "A differential detection scheme for transmit diversity," IEEE J. Select. Areas Commun., vol. 18, pp. 1169 1174 , July 2000.

[2] B. M. Hochwald, and W. Sweldens, "Differential unitary space time modulation,” IEEE Trans. Commun., vol. 48, pp. 2041-2052, Dec. 2000.

[3] B. L. Hughes, "Differential space-time modulation," IEEE Trans. Inform. Theory, vol. 46, pp. 2567-2578, Nov. 2000.

[4] O. Besson and P. Stoica, "On parameter estimation of MIMO flat-fading channels with frequency offsets," IEEE Trans. Signal Processing, vol. 51, pp. 602-613, Mar. 2003.

[5] H. Minn, N. Al-Dhahir, and Y. Li, "Optimal Training Signals for MIMO OFDM Channel Estimation in the Presence of Frequency Offset and Phase Noise," IEEE Trans. Commun., vol. 54, pp. 1754-1759, Oct. 2006.
[6] Z. Liu, G. B. Giannakis, and B. L. Hughes, "Double differential spacetime block coding for time selective fading channels," IEEE Trans. Commun., vol. 49, pp. 1529-1539, Sept. 2001.

[7] X. Ma, "Low complexity block double differential design for OFDM with carrier frequency offset," IEEE Trans. Commun., vol. 53, pp. 21292138, Dec. 2005

[8] T. Cui, F. Gao, A. Nallanathan, and C. Tellambura, "Maximum likelihood detection and optimal code design for differential unitary spacetime modulation with carrier frequency offset", to appear in Proc. IEEE GLOBECOM'07, online http://www.ece-i2r.nus.edu.sg/elegaof.

[9] T. Cui, and C. Tellambura, "Bound-intersection detection for multiplesymbol differential unitary space-time modulation," IEEE Trans. Commun., vol. 53, pp. 2114-2123, Dec. 2005.

[10] — "Multiple-Symbol Differential Detection for Single-Antenna and Multiple-Antenna Systems over Ricean-fading Channels," in Proc, IEEE ICC'06, Istanbul, Turkey, vol. 3, June, 2006, pp. 1439-1444.

[11] R. Schober and L. H. J. Lampe, "Noncoherent receivers for differential space-time modulation," IEEE Trans. Commun., vol. 50, no. 5, pp. 768 - 777, May 2002.

[12] M. O. Damen, K. Abed-Meraim, and J.-C. Belfiore, "Generalised sphere decoder for asymmetrical space-time communication architecture," IEE Elect. Lett., vol. 36, pp. 166-167, Jan. 2000.

[13] I. S. Gradshteny and I. M. Ryzhik, Table of Integrals, Series, and Products. 5th ed, San Diego: Academic Press. 1994.

[14] E. Bach and J. Shallit, Algorithmic Number Theory, Vol. 1: Efficient Algorithms. Cambridge, MA: MIT Press, 1996.

[15] K. L. Clarkson, W. Sweldens, and A. Zheng, "Fast multiple-antenna differential decoding," IEEE Trans. Commun., vol. 49, pp. 253-261, Feb. 2001. 